\title{
Coffee and acute ischemic stroke onset
}

\author{
The Stroke Onset Study \\ (])
}

E. Mostofsky, MPH

G. Schlaug, MD, PhD

K.J. Mukamal, MD,

$\mathrm{MPH}$

W.D. Rosamond, PhD

M.A. Mittleman, MD, $\mathrm{DrPH}$

Address correspondence and reprint requests to Dr. Murray A. Mittleman, Cardiovascular Epidemiology Research Unit, Beth Israel Deaconess Medical Center, 375 Longwood Avenue, Room 423, Boston, MA 02215 mmittlem@bidmc.harvard.edu

\section{ABSTRACT}

Objective: Prior research suggests an acutely elevated risk of myocardial infarction and sudden cardiac death in the hour after coffee intake. However, the risk of ischemic stroke associated with transient exposure to coffee remains unclear. We hypothesized that caffeine intake is associated with a transiently increased risk of ischemic stroke.

Methods: In this multicenter case-crossover study, we interviewed 390 subjects (209 men, 181 women) between January 2001 and November 2006 a median of 3 days after acute ischemic stroke. Each subject's coffee consumption in the hour before stroke symptoms was compared with his or her usual frequency of consumption in the prior year.

Results: Of the 390 subjects, 304 (78\%) drank coffee in the prior year, 232 within 24 hours and 35 within 1 hour of stroke onset. The relative risk (RR) of stroke in the hour after consuming coffee was 2.0 (95\% confidence interval [Cl], 1.4-2.8; $p<0.001)$. There was no apparent increase in risk in the hour following consumption of caffeinated tea ( $R R=0.9,95 \% \mathrm{Cl} 0.4-2.0 ; p=0.85$ ) or cola ( $R R=1.0,95 \% \mathrm{Cl} 0.4-2.4 ; p=0.95)$. The association between ischemic stroke in the hour after coffee consumption was only apparent among those consuming $\leq 1$ cup per day but not for patients who consumed coffee more regularly ( $p$ for trend $=0.002$ ). Relative risks remained similar when the sample was restricted to those who were not simultaneously exposed to other potential triggers and the results remained significant after stratifying by time of day.

Conclusion: Coffee consumption transiently increases the risk of ischemic stroke onset, particularly among infrequent drinkers. Neurology ${ }^{\circledR} 2010 ; 75: 1583-1588$

\section{GLOSSARY}

$\mathbf{C I}=$ confidence interval; $\mathbf{M I}=$ myocardial infarction; $\mathbf{R R}=$ relative risk; TOAST $=$ Trial of Org 10172 in Acute Stroke Treatment.

Approximately 795,000 new or recurrent cases of stroke occur each year. Cerebrovascular disease is a leading cause of serious, long-term disability in the United States and it is the third leading cause of mortality, accounting for approximately 1 of every 17 deaths in the United States in 2005. The estimated direct and indirect cost of stroke for 2009 is $\$ 68.9$ billion. ${ }^{1}$

Coffee has been documented to have acute deleterious physiologic effects within hours after consumption, including elevated levels of (nor)epinephrine, ${ }^{2}$ increased systolic and diastolic blood pressure ${ }^{3}$ and vascular resistance, ${ }^{4}$ and a slight drop in heart rate. ${ }^{3}$ Compared to decaffeinated coffee, consumption of caffeinated coffee acutely increases arterial stiffness ${ }^{5,6}$ and impairs endothelium-dependent vasodilation. ${ }^{7}$ Caffeine has also been shown to eliminate the protective effect of ischemic preconditioning. ${ }^{8}$ There is evidence of an acutely elevated risk of acute myocardial infarction ${ }^{9}$ and sudden cardiac death ${ }^{10}$ in the hour after coffee intake, but to our knowledge, there has not been any research to examine the risk of ischemic stroke associated with transient exposure to caffeine and whether the risk varies by the sources of caffeine.

e-Pub ahead of print on September 29, 2010, at www.neurology.org.

From the Department of Medicine (E.M., K.J.M., M.A.M.), Department of Neurology (G.S.), and Division of General Medicine \& Primary Care (K.J.M.), Cardiovascular Epidemiology Research Unit, Beth Israel Deaconess Medical Center, Harvard Medical School, Boston; Department of Epidemiology (E.M., M.A.M.), Harvard School of Public Health, Boston, MA; and Department of Epidemiology (W.D.R.), University of North Carolina School of Public Health, Chapel Hill.

Study funding: Supported by the American Heart Association (0140219N to M.A.M.) and the NIH/NIAID (T32-A1007535-11 to E.M.).

Disclosure: Author disclosures are provided at the end of the article. 
Our primary objective was to examine the association between caffeinated coffee consumption and the risk of acute ischemic stroke in the subsequent hour.

METHODS Study design. The Stroke Onset Study used a case-crossover design, a variation of a case-control design that is appropriate when a brief exposure (caffeinated coffee consumption) causes a transient change in the risk of an acute outcome (ischemic stroke). ${ }^{11,12}$ We compared a subject's coffee consumption in the hour prior to onset of stroke symptoms (the case period) with the same subject's usual frequency of coffee consumption in the prior year (the control period). Because control information for each subject is based on his or her own past exposure experience, self-matching eliminates confounding by risk factors that are constant within individuals over the sampling period but often differ between study subjects.

Study population. The Stroke Onset Study was conducted in 3 medical centers (Beth Israel Deaconess Medical Center, Boston, MA; University of North Carolina Hospitals, Chapel Hill, NC; Vancouver Island Health Authority, Victoria, BC) between January 2001 and November 2006. Trained research staff identified eligible subjects by reviewing admission logs and charts of subjects admitted to each hospital's stroke service. Additionally, subjects with new onset of an acute neurologic syndrome compatible with stroke were screened upon admission to emergency departments. Presumed stroke etiology was determined according to an abbreviated Trial of Org 10172 in Acute Stroke Treatment (TOAST) classification system. ${ }^{13}$ Across all sites, $43 \%$ of patients with confirmed ischemic stroke met all inclusion criteria. Of these, $83 \%$ agreed to participate, $5.5 \%$ refused, and the remaining $12.5 \%$ were discharged from the hospital before the interviewers were able to approach them. The final sample includes 390 subjects (209 men and 181 women) who were interviewed a median of 3 days (range $0-14$ ) after sustaining an acute ischemic stroke.

For each case, trained study personnel using standardized abstraction forms recorded data on demographics, medical history, and admission laboratory results. To be eligible, subjects were required to have a neurologist-confirmed diagnosis of acute ischemic stroke, either by clinical diagnosis or appropriate imaging studies, to be English-speaking, and to be free of dementia prior to the index event. Subjects were excluded if they were unable to complete the structured interview because they were cognitively impaired, had poor memory around the time of the stroke, could not identify the time of onset of their stroke symptoms, experienced aphasia, or were too ill to complete the structured interview that lasted 30 to 45 minutes.

Coffee consumption. Interviewers used a structured questionnaire and asked subjects if they had consumed any coffee in the year preceding their stroke. Subjects who reported any coffee consumption were also asked to report the last time that they had consumed a serving of coffee and their usual frequency of coffee consumption over the prior year. The portion size for 1 serving of coffee was defined as 8 ounces or 1 cup of caffeinated coffee. Similar questions were asked about consumption of caffeinated tea and cola. Subjects were also asked to report the timing of their last exposure to other potential triggers and usual frequency of these factors in the year preceding the onset of stroke symptoms. Other information collected from the interview included medication use and symptoms on the day of the stroke.
Reliability of the questionnaire. The test-retest reliability of the Stroke Onset Study questionnaire was assessed in a subgroup of 25 subjects who were re-interviewed up to 6 days after their initial interview and asked again about their coffee consumption patterns in the past year as well as the time of their last drink before the onset of their stroke symptoms. The intraclass correlation for the usual frequency of coffee consumption was found to be excellent (0.96), and there was high agreement for reporting of any coffee consumption during the past year $(\kappa=$ $0.96)$ and during the hour preceding stroke onset $(\kappa=0.88)$.

Standard protocol approvals, registrations, and patient consents. The protocol was approved by the institutional review board at each participating center and written informed consent was obtained from all subjects participating in the study.

Statistical analysis. Each subject in a case-crossover study forms his or her own stratum and thus is his or her own control. ${ }^{11,12}$ The ratio of the observed exposure frequency in the case period to the expected frequency based on control information about coffee consumption in the previous year was used to calculate estimates of the rate ratio as a measure of relative risk (RR). We multiplied the usual annual frequency of coffee consumption by the hypothesized window of its physiologic effect (1 hour in the primary analysis) to estimate the amount of person-time exposed to coffee. The unexposed person-time was calculated by subtracting this value from the number of hours in 1 year. The data were analyzed using methods for cohort studies with sparse data in each stratum. Similar analyses were conducted for caffeinated tea and caffeinated cola.

To estimate the length of time from coffee consumption to the onset of ischemic stroke, RRs were calculated for each 1-hour period before stroke onset. We stratified by sex, age $(<65,65-$ 75 , and $>75$ years), smoking status (never, former, current), habitual moderate or vigorous physical activity (3 or more times per week vs fewer than 3 times per week), and stroke etiology and compared the RRs by means of a test for homogeneity. ${ }^{14}$ Thirty-two subjects were excluded from the analysis stratified by stroke etiology because it was not determined at 1 center. We stratified by intake of coffee in the past week (up to 1 cup per day, more than 1 to 3 cups per day, more than 3 cups per day) and conducted a test for trend. ${ }^{15}$ Because there is a circadian peak of stroke onset in the morning hours ${ }^{16}$ and people tend to drink more coffee in the morning than during the rest of the day, we conducted a sensitivity analysis which we restricted to subjects experiencing strokes between 6 AM and $9 \mathrm{AM}$. We made the conservative assumption that all coffee in the past year is only consumed during that time of day, which should result in a conservative estimate of the RR. To evaluate whether potential triggers could account for the observed association, we conducted a sensitivity analysis excluding subjects simultaneously exposed to other potential triggers (e.g., vigorous physical exertion, alcohol, marijuana, and anger) in the hour preceding their stroke and a sensitivity analysis excluding subjects reporting a stroke or myocardial infarction (MI) within the year preceding the index event. In another sensitivity analysis, we used the number of servings of coffee consumed in the week preceding the stroke as the control information. All reported $p$ values are 2-sided.

RESULTS The characteristics of the Stroke Onset Study subjects are presented in the table. Of the 390 subjects with acute ischemic stroke who were interviewed, 305 (78\%) reported that they had consumed 


\begin{tabular}{|c|c|c|c|}
\hline \multirow[t]{2}{*}{ Table } & es of the stroke o & study populat & \multirow[b]{2}{*}{$p$ Value } \\
\hline & $\begin{array}{l}\text { Coffee drinkers } \\
(n=304)\end{array}$ & $\begin{array}{l}\text { Nondrinkers } \\
(\mathrm{n}=85)\end{array}$ & \\
\hline Age, y & $68.1 \pm 14.3$ & $70.0 \pm 13.9$ & 0.26 \\
\hline Men & $169(56)$ & $39(46)$ & 0.11 \\
\hline \multicolumn{2}{|l|}{ Smoking status } & & $<0.0001$ \\
\hline Never & $84(28)$ & $44(52)$ & \\
\hline Former & $152(50)$ & 33 (39) & \\
\hline Current & $68(22)$ & $8(10)$ & \\
\hline $\begin{array}{l}\text { Obesity (body mass index } \\
\left.\geq 30 \mathrm{~kg} / \mathrm{m}^{2}\right)^{\mathrm{b}}\end{array}$ & $73(25)$ & $16(19)$ & 0.30 \\
\hline Diabetes & $75(25)$ & 19 (22) & 0.66 \\
\hline Hypercholesterolemia & $117(38)$ & $29(34)$ & 0.46 \\
\hline Hypertension & $197(65)$ & $55(65)$ & 0.99 \\
\hline Atrial fibrillation & $34(11)$ & $20(24)$ & 0.004 \\
\hline \multicolumn{4}{|l|}{ History of: } \\
\hline Myocardial infarction & $39(13)$ & $10(12)$ & 0.79 \\
\hline Stroke & $60(20)$ & $13(15)$ & 0.35 \\
\hline TIA & $38(13)$ & $9(11)$ & 0.63 \\
\hline Coronary revascularization & $29(10)$ & $12(14)$ & 0.22 \\
\hline Carotid endarterectomy & $7(2)$ & $0(0)$ & 0.16 \\
\hline \multicolumn{2}{|l|}{ Stroke etiology ${ }^{c}$} & & 0.52 \\
\hline Small vessel & 88 (32) & $23(30)$ & \\
\hline Large vessel & $55(20)$ & $12(16)$ & \\
\hline Cardioembolic & $58(21)$ & $22(29)$ & \\
\hline Other/undetermined & $79(28)$ & $20(26)$ & \\
\hline
\end{tabular}

a Values are mean $\pm \mathrm{SD}$ or $\mathrm{n}(\%)$.

b There were no data available on body mass index for 7 subjects.

${ }^{\mathrm{c}}$ At one of the centers, stroke etiology was not determined $(\mathrm{n}=32)$.

coffee in the prior year. One subject provided no information about usual coffee intake, so the subject was excluded from all analyses. Compared to nondrinkers, subjects who reported coffee consumption within the past year were more likely to be current

\section{Figure 1 Relative risk of ischemic stroke within 1 hour according to the beverage type}

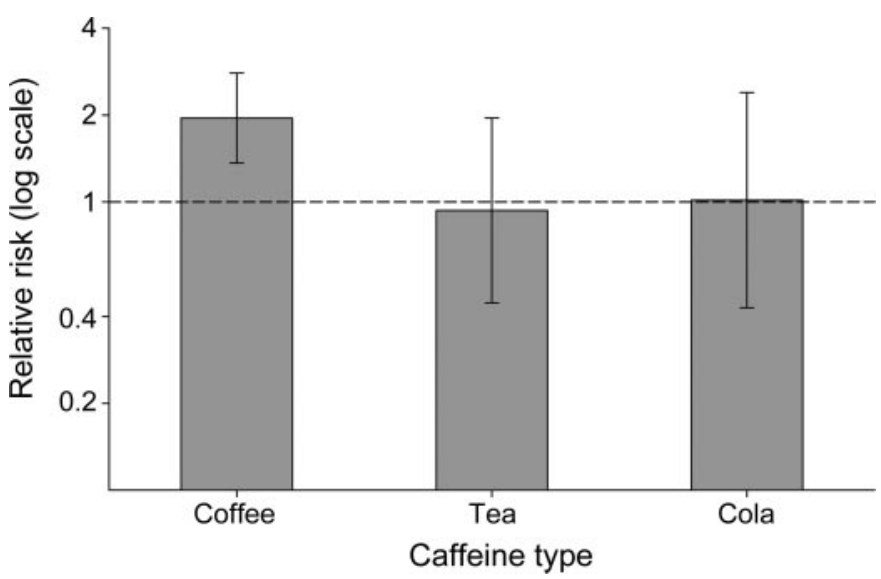

The error bars indicate the $95 \%$ confidence limits. The dashed line indicates the baseline risk. smokers and they were less likely to have a history of atrial fibrillation. Among the 304 subjects who drank coffee in the prior year, $249(82 \%)$ drank at least 1 serving of coffee per day, $35(12 \%)$ drank at least once per week, 13 (4\%) drank coffee at least once per month, and 7 (2\%) drank coffee less than once per month; the median frequency of consumption was 7 times per week. Thus, the average amount of persontime exposed to coffee in the prior year (the control period) was 364 hours per subject.

Among the 304 subjects who drank coffee in the prior year, 35 subjects consumed at least 1 serving of coffee within 1 hour of the onset of stroke symptoms (the case period). We found that within 1 hour after coffee consumption, the risk of stroke onset was 2.0fold higher (95\% confidence interval [CI] 1.4-2.8; $p<0.001)$ compared with periods of nonuse, and it rapidly returned to baseline thereafter. The risks of ischemic stroke in the hour following consumption of caffeinated tea $(\mathrm{RR}=0.9,95 \%$ CI $0.4-2.0 ; p=$ $0.85)$ or cola $(\mathrm{RR}=1.0,95 \%$ CI $0.4-2.4 ; p=0.95)$ were not elevated (figure 1 ). The RRs for coffee consumption did not vary by sex $(p=0.13)$, age ( $p=$ $0.56)$, smoking status $(p=0.22)$, physical activity ( $p=0.44)$, or stroke etiology ( $p=0.29)$. When we stratified by daily intake of caffeinated coffee in the past week, the association between ischemic stroke in the hour after coffee consumption was only apparent among those consuming 1 or fewer cups per day but not for patients who consumed coffee more regularly ( $p$ for trend $=0.002$; figure 2 ).

In a sensitivity analysis using the conservative assumption that all coffee in the past year is only consumed in the morning (6 AM-9 AM), coffee consumption was still associated with an increased risk of ischemic stroke onset $(\mathrm{RR}=3.8,95 \% \mathrm{CI}$ $2.0-7.0 ; p<0.001)$. Among the 304 subjects exposed to coffee in the prior year, 75 subjects were exposed to other potential triggers in the hour prior to stroke onset. Of the 35 people exposed to coffee in the hour prior to stroke onset, 3 were also exposed to vigorous physical activity, 1 experienced feelings of anger, 1 smoked a cigarette, and 1 drank an alcoholic beverage. When we conducted an analysis excluding the 75 people exposed to any potential stroke trigger in the hour preceding stroke onset, the results remained similar. RRs were similar when the sample was restricted to those who did not experience a stroke or MI in the prior year $(\mathrm{n}=283)$. On average, subjects reported almost identical levels of coffee consumption in the week preceding the stroke and in the prior year, so the RR did not change when we used each subject's reported frequency of consumption in the past week to estimate control periods. 


\section{Figure 2 Relative risk of ischemic stroke within 1 hour by drinking frequency in the prior week}

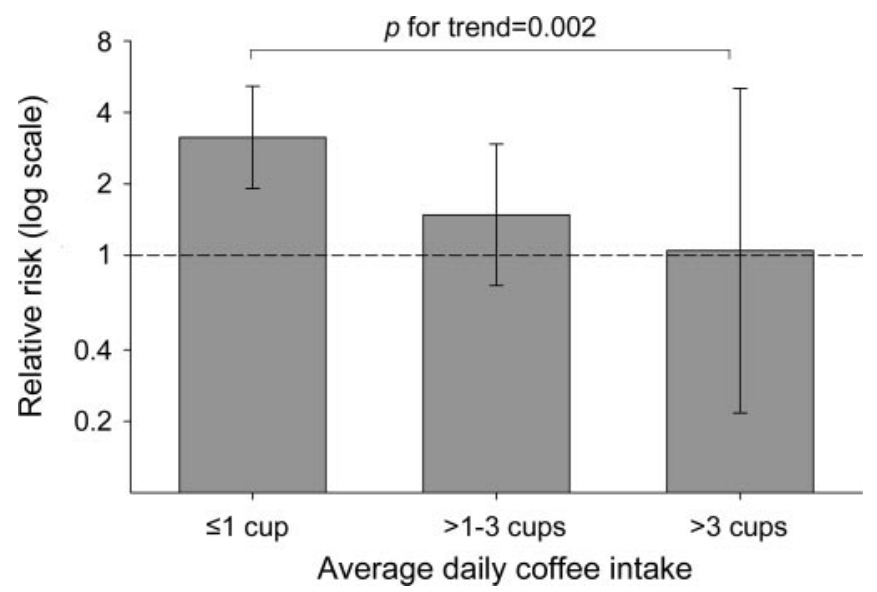

The error bars indicate the $95 \%$ confidence limits. The dashed line indicates the baseline risk.

DISCUSSION In this study, we found that coffee consumption was associated with a transient risk of ischemic stroke in the subsequent hour that was 2.0 times higher than the risk during periods with no coffee consumption, and the risk returned to baseline by 2 hours. Our findings are consistent with 2 casecrossover studies on coffee intake and other acute cardiovascular events. ${ }^{9,10}$ In a study of 503 incident cases of nonfatal myocardial infarction, ${ }^{9}$ the RR of acute myocardial infarction in the hour after coffee intake was 1.5 (95\% CI 1.2-1.9), and the association was particularly strong for those who are only occasional coffee drinkers, those with 3 or more cardiovascular risk factors, and those who lead sedentary lifestyles. A similar study ${ }^{10}$ reported that the RR of dying from sudden cardiac death within an hour after drinking a cup of coffee was $73 \%$ higher $(95 \%$ CI 1.1-2.7) than periods with no coffee consumption. Similar to the prior study of myocardial infarction, ${ }^{9}$ we found that the effect of coffee consumption was not significantly different in men vs women, subjects older or younger than 65 years of age, or by smoking status, frequency of habitual physical activity, or stroke etiology.

We did not find an association between consumption of caffeinated tea or cola and ischemic stroke. The lower dose of caffeine in these beverages may account for this difference. ${ }^{17}$

Evidence from experimental studies indicates that coffee acutely increases circulating concentrations of (nor)epinephrine, ${ }^{2}$ raises systolic and diastolic blood pressure, ${ }^{3}$ increases arterial stiffness, ${ }^{5,6}$ and impairs endothelium-dependent vasodilation. ${ }^{7}$ Some studies have shown that habitual consumption results in a tolerance to the acute effects of caffeine, ${ }^{18,19}$ while others have found that some individuals continue to experience these acute effects with each drinking episode $^{20,21}$ and that the tolerance disappears after 12 hours of abstinence. ${ }^{22}$ This is consistent with our findings and others, ${ }^{9}$ indicating that the RR of ischemic stroke in the hour following consumption of caffeinated coffee was higher among people reporting lower levels of habitual coffee consumption.

There have been discrepant findings on the association between habitual coffee intake and ischemic stroke. One study found that total coffee consumption was not associated with an increased stroke risk in men ${ }^{23}$ and another study found that coffee consumption was associated with an increased risk of ischemic stroke among hypertensive men who reported no previous or current smoking. ${ }^{24}$ However, others have reported that habitual coffee consumption is associated with a lower risk of ischemic stroke. In the Alpha-Tocopherol, Beta-Carotene Cancer Prevention Study of men, smokers who drank 8 or more cups of coffee per day had a $23 \%$ lower risk of stroke than those who drank fewer than 2 cups per day. ${ }^{25}$ Similarly, in the Nurses' Health Study, women who drank 4 or more cups of coffee per day had a $20 \%$ lower risk of stroke than those who did not consume coffee. ${ }^{26}$ These results are concordant with our finding of no increased risk among those who drink coffee regularly, suggesting that habitual consumption results in a tolerance to the acute effects of caffeine. ${ }^{18,19}$

There are some limitations to our study that warrant discussion. Based on previous research, our a priori hypothesis was focused on caffeinated coffee consumption so we did not collect information on the coffee type or preparation method or on consumption of decaffeinated beverages. Since the casecrossover design uses subjects as their own controls, there is no variability in traditional risk factors for stroke. ${ }^{11}$ However, confounding by factors that change over time within individual subjects can occur if other transient risk factors occur during the case period of interest. In a sensitivity analysis using a conservative assumption that all coffee in the past year was consumed in the morning, the results remained significant. Though there may be residual confounding by time of day, this suggests that the findings are not fully explained by the co-occurrence of coffee and the circadian peak in stroke onset. A sensitivity analysis excluding subjects reporting other potential triggers in the hour preceding stroke onset did not materially alter the results. We had limited power to determine whether the effect of coffee consumption varied by clinical characteristics or stroke etiology.

Recall bias may occur if there is systematic error in reporting usual exposure frequency compared 
with exposure during the hour before stroke onset. ${ }^{11}$ If subjects overestimate exposure in the period immediately before the stroke and underestimate exposure in the past year, the RR may be biased upwards. In an effort to minimize reporting bias and to maintain subject confidentiality, efforts were made to ensure the subject's privacy during the interview. We used a standardized structured interview and subjects were not informed of the duration of the hypothesized case period. If patients whose strokes were triggered by coffee consumption were more likely to participate or had milder strokes than those whose strokes were unrelated to coffee, the apparent RR might be overestimated. The patients we interviewed tended to have milder strokes, as those with aphasia, memory difficulties, or severe strokes leaving them incapacitated or too ill to be interviewed were excluded from the study. Therefore, our results may not be generalizable to subjects presenting with a severe or fatal stroke. However, the RR for recent coffee consumption did not vary by stroke etiology, suggesting that the role of coffee as a stroke trigger does not differ by stroke mechanism and is unlikely to vary by severity.

\section{AUTHOR CONTRIBUTIONS}

Statistical analysis was conducted by E. Mostofsky and Dr. Mittleman.

\section{ACKNOWLEDGMENT}

The authors thank Cindy Aiello for administrative assistance.

\section{DISCLOSURE}

E. Mostofsky receives research support from the NIH/NIAID (T32A1007535-11). Dr. Schlaug receives research support from the NIH/ NIDCD (1RO1 DC008796 [PI], R01 DC009823-01[PI], and 3R01DC008796-02S1 [PI]). Dr. Mukamal serves on the editorial board of Nutrition; has received institutional research support from Martek Corporation; and receives research support from the NIH (HL094555 [PI], HL091874 [PI], and AA016110 [PI]) and from Harvard Medical School. Dr. Rosamond receives research support from the CDC (NCDHHS 01602-05 [subcontract, PI]) and the NIH (N01-HC-55015 [coinvestigator]). Dr. Mittleman served on the editorial board of Epidemiology and serves on the editorial board of Environmental Health Perspectives; and has received/receives research support from the NIH (ES009825 [Project PI], ES017125 [PI], AA016567 [PI], AA016110 [coinvestigator], HL091874 [coinvestigator], DK071083 [coinvestigator], and UL1RR025758 [coinvestigator]), the $\mathrm{CDC} / \mathrm{NIOSH}$, and the American Heart Association.

Received February 17, 2010. Accepted in final form June 1, 2010.

\section{REFERENCES}

1. Lloyd-Jones D, Adams R, Carnethon M, et al. Heart disease and stroke statistics-2009 update: a report from the American Heart Association Statistics Committee and Stroke Statistics Subcommittee. Circulation 2009;119: 480-486.

2. Smits P, Pieters G, Thien T. The role of epinephrine in the circulatory effects of coffee. Clin Pharmacol Ther 1986;40: 431-437.

3. Nurminen ML, Niittynen L, Korpela R, Vapaatalo H. Coffee, caffeine and blood pressure: a critical review. Eur J Clin Nutr 1999;53:831-839.
4. Mahmud A, Feely J. Acute effect of caffeine on arterial stiffness and aortic pressure waveform. Hypertension 2001;38:227-231.

5. Vlachopoulos C, Kosmopoulou F, Panagiotakos D, et al. Smoking and caffeine have a synergistic detrimental effect on aortic stiffness and wave reflections. J Am Coll Cardiol 2004;44:1911-1917.

6. Karatzis E, Papaioannou TG, Aznaouridis K, et al. Acute effects of caffeine on blood pressure and wave reflections in healthy subjects: should we consider monitoring central blood pressure? Int J Cardiol 2005;98:425-430.

7. Papamichael CM, Aznaouridis KA, Karatzis EN, et al. Effect of coffee on endothelial function in healthy subjects: the role of caffeine. Clin Sci 2005;109:55-60.

8. Riksen NP, Zhou Z, Oyen WJ, et al. Caffeine prevents protection in two human models of ischemic preconditioning. J Am Coll Cardiol 2006;48:700-707.

9. Baylin A, Hernandez-Diaz S, Kabagambe EK, Siles X, Campos $\mathrm{H}$. Transient exposure to coffee as a trigger of a first nonfatal myocardial infarction. Epidemiology 2006; 17:506-511.

10. Selb Semerl J, Selb K. Coffee and alcohol consumption as triggering factors for sudden cardiac death: case-crossover study. Croat Med J 2004;45:775-780.

11. Maclure M. The case-crossover design: a method for studying transient effects on the risk of acute events. Am J Epidemiol 1991;133:144-153.

12. Mittleman MA, Maclure M, Robins JM. Control sampling strategies for case-crossover studies: an assessment of relative efficiency. Am J Epidemiol 1995;142:91-98.

13. Adams HP Jr, Bendixen BH, Kappelle LJ, et al. Classification of subtype of acute ischemic stroke: definitions for use in a multicenter clinical trial: TOAST: Trial of Org 10172 in Acute Stroke Treatment. Stroke 1993;24:35-41.

14. Rothman KJ, Greenland S, Lash TL. Modern Epidemiology, 3rd ed. Philadelphia: Lippincott Williams \& Wilkins; 2008.

15. Berlin JA, Longnecker MP, Greenland S. Meta-analysis of epidemiologic dose-response data. Epidemiology 1993;4: $218-228$

16. Argentino $\mathrm{C}$, Toni $\mathrm{D}$, Rasura $\mathrm{M}$, et al. Circadian variation in the frequency of ischemic stroke. Stroke 1990;21:387389.

17. Gilbert RM, Marshman JA, Schwieder M, Berg R. Caffeine content of beverages as consumed. Can Med Assoc J 1976;114:205-208.

18. Robertson D, Frolich JC, Carr RK, et al. Effects of caffeine on plasma renin activity, catecholamines and blood pressure. N Engl J Med 1978;298:181-186.

19. Ammon HP, Bieck PR, Mandalaz D, Verspohl EJ. Adaptation of blood pressure to continuous heavy coffee drinking in young volunteers: a double-blind crossover study. Br J Clin Pharmacol 1983;15:701-706.

20. Lovallo WR, Wilson MF, Vincent AS, Sung BH, McKey BS, Whitsett TL. Blood pressure response to caffeine shows incomplete tolerance after short-term regular consumption. Hypertension 2004;43:760-765.

21. Farag NH, Vincent AS, McKey BS, Whitsett TL, Lovallo WR. Hemodynamic mechanisms underlying the incomplete tolerance to caffeine's pressor effects. Am J Cardiol 2005;95:1389-1392.

22. Watson J, Deary I, Kerr D. Central and peripheral effects of sustained caffeine use: tolerance is incomplete. $\mathrm{Br} \mathrm{J}$ Clin Pharmacol 2002;54:400-406. 
23. Grobbee DE, Rimm EB, Giovannucci E, Colditz G, Stampfer M, Willett W. Coffee, caffeine, and cardiovascular disease in men. N Engl J Med 1990;323:1026-1032.

24. Hakim AA, Ross GW, Curb JD, et al. Coffee consumption in hypertensive men in older middle-age and the risk of stroke: the Honolulu Heart Program. J Clin Epidemiol 1998;51:487-494.
25. Larsson SC, Mannisto S, Virtanen MJ, Kontto J, Albanes D, Virtamo J. Coffee and tea consumption and risk of stroke subtypes in male smokers. Stroke 2008;39:1681-1687.

26. Lopez-Garcia E, Rodriguez-Artalejo F, Rexrode KM, Logroscino G, Hu FB, van Dam RM. Coffee consumption and risk of stroke in women. Circulation 2009;119: $1116-1123$.

\section{NeuroSAE IIITM}

\section{Assess your knowledge of neurology.}

Discover your strengths and areas in need of improvement. Compare your performance to other neurologists. Take the necessary steps towards fulfilling the self-assessment component of the American Board of Psychiatry and Neurology (ABPN)-mandated Maintenance of Certification (MOC) requirements.

The new NeuroSAE IIITM has new questions and more images. It is a convenient way to get the practice you need for fulfilling your MOC requirements. www.aan.com/neurosae 